\title{
Effects of Hydrochlorothiazide on Oxidative Stress and Pulse Pressure in Hypertensive Patients with Chronic Stroke: The EMINENT Study
}

\author{
Hiroshi Nakane ${ }^{1}$, Masahiro Kamouchi ${ }^{2-4}$, Jun Hata ${ }^{2,4,5}$, Setsuro Ibayashi ${ }^{6}$, Kenji Kusuda ${ }^{6}$, \\ Tsuyoshi Omae ${ }^{7}$, Tetsuhiko Nagao ${ }^{8}$, Tetsuro Ago ${ }^{2,5}$ and Takanari Kitazono ${ }^{2,4,5}$; \\ for the EMINENT Study Investigators
}

\begin{abstract}
Objective Thiazide diuretics are reported to have antioxidant effects and reduce pulse pressure (PP). The aim of this study was to elucidate whether hydrochlorothiazide additionally exerts such effects in stroke patients under treatment with losartan.

Methods This study was an open-label, randomized, multicenter study. Patients with a history of chronic stroke and treatment with angiotensin receptor blockers or angiotensin-converting enzyme inhibitors for essential hypertension were enrolled. Fifty-five hypertensive patients were randomly assigned to two groups: those further treated with hydrochlorothiazide and those further treated with non-diuretic antihypertensive drugs.

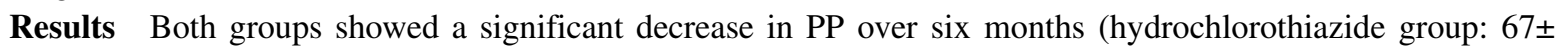
$12 \mathrm{mmHg}$ to $58 \pm 12, \mathrm{p}<0.001$; non-diuretic group: $72 \pm 12$ to $61 \pm 12, \mathrm{p}<0.001$ ), although no significant differences were observed between the two groups. The malondialdehyde-modified low-density lipoprotein levels did not change significantly after treatment in either group.

Conclusion In this study, hydrochlorothiazide treatment did not provide any additional benefits over nondiuretic antihypertensive drugs in terms of antioxidant effects or reducing PP.
\end{abstract}

Key words: angiotensin receptor blocker, stroke, thiazide, oxidative stress, pulse pressure

(Intern Med 54: 573-577, 2015)

(DOI: 10.2169/internalmedicine.54.2631)

\section{Introduction}

Thiazide diuretics have been shown to reduce cardiovascular events in clinical trials $(1,2)$. In addition to their blood pressure-lowering actions, the antioxidant effects of these agents may have a beneficial impact on the incidence of cardiovascular events $(3,4)$. Furthermore, thiazide diuret- ics have been reported to reduce pulse pressure (PP) $(5,6)$, which may also help to prevent cardiovascular diseases. Based on these findings, thiazide diuretics are useful for preventing cardiovascular events in hypertensive patients who suffer from stroke. However, the prescription of diuretics is less prevalent in such patients in Japan due to concerns regarding the adverse metabolic effects of these drugs.

Angiotensin receptor blockers (ARBs) may have favorable

${ }^{1}$ Cerebrovascular and Neurology Center, Cerebrovascular Division, National Hospital Organization Fukuoka Higashi Medical Center, Japan, ${ }^{2}$ Department of Nephrology, Hypertension, and Strokology, Kyushu University Hospital, Japan, ${ }^{3}$ Department of Health Care Administration and Management, Graduate School of Medical Sciences, Kyushu University, Japan, ${ }^{4}$ Center for Cohort Studies, Graduate School of Medical Sciences, Kyushu University, Japan, ${ }^{5}$ Department of Medicine and Clinical Science, Graduate School of Medical Sciences, Kyushu University, Japan, ${ }^{6}$ Department of Medicine, Seiai Rehabilitation Hospital, Japan, ${ }^{7}$ Department of Medicine, Imazu Red Cross Hospital, Japan and ${ }^{8}$ Midorino Clinic, Japan

Received for publication February 5, 2014; Accepted for publication August 27, 2014

Correspondence to Dr. Hiroshi Nakane, nakaneh@fukuokae2.hosp.go.jp 
pleomorphic effects in patients with stroke, including vasodilation of the cerebral arteries, anti-inflammatory effects and improvements in insulin resistance $(7,8)$. Therefore, ARBs are currently used in a large number of Japanese hypertensive patients with stroke as antihypertensive agents. The concomitant use of a thiazide diuretic with an ARB is beneficial for reducing blood pressure (BP) $(9,10)$, as thiazide diuretics have complementary actions to ARBs (9). However, it remains unclear whether diuretics show more potent antioxidant effects or achieve a more significant reduction in PP compared with other non-diuretic antihypertensive drugs when administered in addition to ARBs.

In order to evaluate the antioxidant and PP-reducing effects of thiazide diuretics, we performed an open-label, randomized, multicenter study, the Effects of sodium Management on the PP and oxidative stress IN hypertensive patiENTs with chronic stroke (EMINENT) study. The aim of this study was to elucidate whether thiazide, administered in combination with an ARB, exerts more prominent antioxidant effects or more remarkably reduces PP without adverse effects in patients with a previous history of stroke.

\section{Materials and Methods}

\section{Study subjects}

This study was conducted at four hospitals participating in the EMINENT study group: Kyushu University Hospital, National Hospital Organization Fukuoka Higashi Medical Center, Seiai Rehabilitation Hospital and Imazu Red Cross Hospital. Patients who satisfied the following criteria were prospectively enrolled: an age between 20 and 75 years, a history of previous stroke occurring more than three months before registration and treatment with $\mathrm{ARBs}$ or angiotensinconverting enzyme inhibitors (ACEIs) for essential hypertension. The exclusion criteria were as follows: congestive heart failure (New York Heart Association class 2 or higher), a serum creatinine level of $>2.0 \mathrm{mg} / \mathrm{dL}$, a HbA1c level of $>8.0 \%$, an alanine aminotransferase (ALT) level more than three times the normal upper limit, cardiovascular diseases, including stroke or myocardial infarction, diagnosed within three months before registration and current treatment with diuretics. Each institutional ethics committee approved the study protocol. Written informed consent was obtained from all subjects prior to enrollment.

\section{Study protocol}

The present study was conducted in accordance with the principles of the Declaration of Helsinki. This open-label, randomized, multicenter study consisted of a two-month screening period and subsequent six-month treatment period. At the beginning of the screening period, the prescribed ARB or ACEI was changed to treatment with $50 \mathrm{mg} /$ day of losartan. At least two BP measurements were obtained over two months during the screening period. Patients whose baseline BP measurements remained over 140/90 mmHg af- ter the screening period were randomly assigned to two groups: those in whom losartan was changed to a combination of fixed-dose losartan $(50 \mathrm{mg})$ and hydrochlorothiazide (HCTZ, $12.5 \mathrm{mg}$ ) (diuretic group), and those in whom an antihypertensive drug other than an ARB, ACEI or diuretic was additionally administered (non-diuretic group). All antihypertensive drugs were allowed, except for ARBs, ACEIs and diuretics, when the BP exceeded 140/90 mmHg. The subjects' clinical symptoms, sitting BP, pulse rate and serum parameters, including the levels of potassium, uric acid, lipids, creatinine, glucose, $\mathrm{HbA} 1_{c}$, high-sensitivity C-reactive protein (hs-CRP) and malondialdehyde-modified low-density lipoprotein (MDA-LDL), were evaluated three times, i.e., during the screening period and at the beginning and end of the treatment period.

Sixty-four patients were enrolled in this study. Among them, six patients refused to participate and two patients were excluded because their BP values declined to under $140 / 90 \mathrm{mmHg}$ during the screening period. The remaining 56 patients were randomized to the two groups: 29 patients were assigned to the diuretic group and 27 patients were assigned to the non-diuretic group. We were unable to provide follow-up for one patient in the diuretic group because he did not return to the hospital. Ultimately, 55 patients (28 in the diuretic group and 27 in the non-diuretic group) were included in the analysis (Figure).

\section{Statistical analysis}

All data are expressed as the mean \pm SD. The statistical analyses were performed using Student's $t$-test for continuous parameters and the chi-square test for categorical variables. Statistical significance was assumed for values of $\mathrm{p}<$ 0.05 . The hs-CRP levels were changed to logarithmic values because they were not normally distributed.

\section{Results}

The baseline characteristics of the study population were summarized in Table 1. There were no significant differences in age, sex or the incidence of diabetes or dyslipidemia between the two groups. The systolic blood pressure (SBP), diastolic blood pressure (DBP), PP and heart rate values obtained after randomization were also not significantly different between the groups. Calcium antagonists were prescribed more often after randomization in the nondiuretic group.

The laboratory data at six months were not significantly different from those observed at baseline, except for the uric acid and HbA1c levels, both of which significantly increased at six months, although they did not exceed the upper normal limit in the diuretic group (Table 2). Meanwhile, the SBP values in the both groups and the DBP values in the diuretic group significantly decreased, whereas the DBP values in the non-diuretic group did not, from baseline to six months after randomization. The changes in the SBP and DBP values were not significantly different between the two 


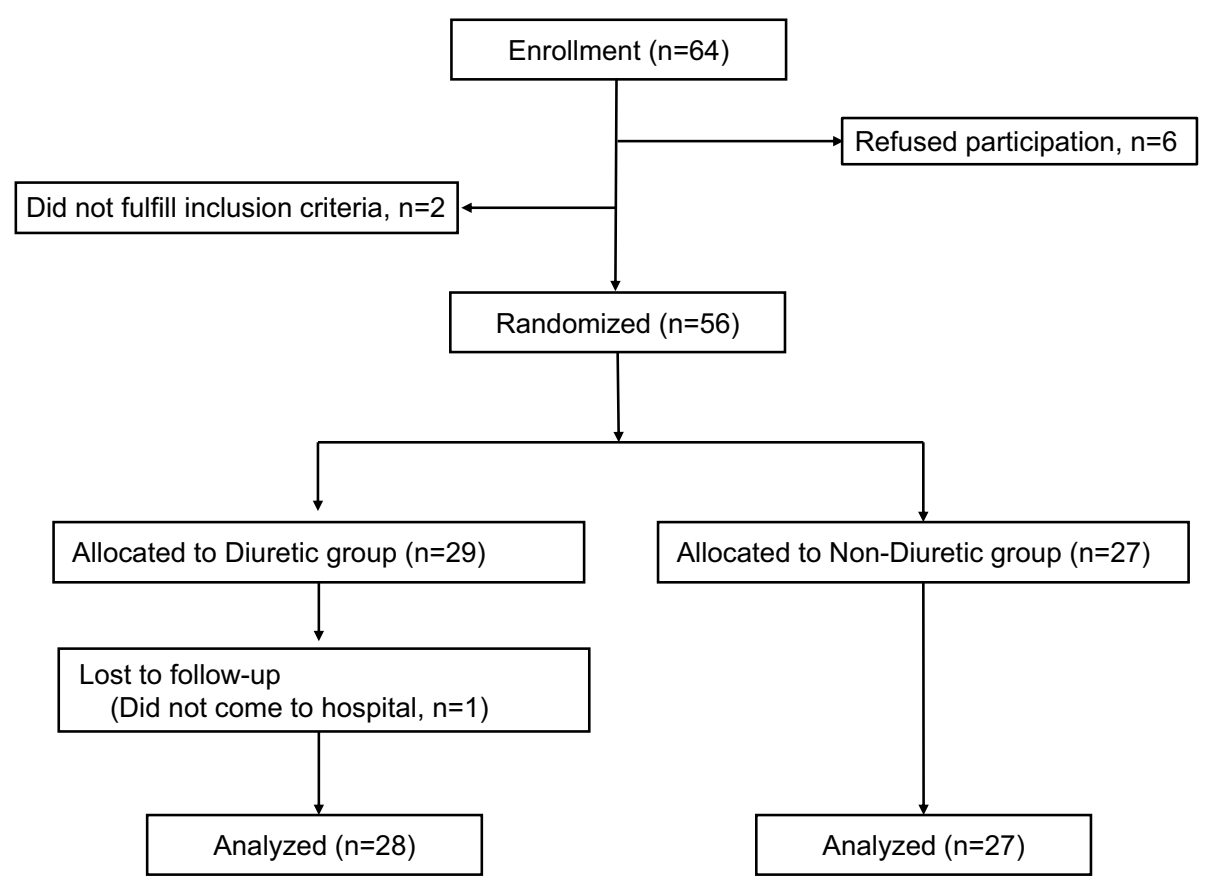

Figure. Trial profile

Table 1. Baseline Characteristics in the Diuretic and Nondiuretic Groups

\begin{tabular}{|c|c|c|c|}
\hline & $\begin{array}{c}\text { Diuretic group } \\
n=28\end{array}$ & $\begin{array}{c}\text { Non-diuretic group } \\
n=27\end{array}$ & $\mathrm{p}$ \\
\hline$\overline{\text { Age, } y}$ & $64 \pm 10$ & $63 \pm 8$ & 0.922 \\
\hline Male & $23(82 \%)$ & $17(63 \%)$ & 0.196 \\
\hline Diabetes mellitus & $4(14 \%)$ & $9(33 \%)$ & 0.179 \\
\hline Dyslipidemia & $9(32 \%)$ & $13(48 \%)$ & 0.349 \\
\hline \multicolumn{4}{|c|}{ Antihypertensives with losartan after randomization } \\
\hline Calcium antagonists & $18(64 \%)$ & $25(93 \%)$ & 0.027 \\
\hline$\beta$-blockers & $4(14 \%)$ & $1(4 \%)$ & 0.370 \\
\hline$\alpha \beta$-blockers & $1(4 \%)$ & $3(11 \%)$ & 0.577 \\
\hline$\alpha$-blockers & $1(4 \%)$ & $4(15 \%)$ & 0.327 \\
\hline BMI, $\mathrm{kg} / \mathrm{m}^{2}$ & $25.5 \pm 4.7$ & $24.2 \pm 3.2$ & 0.240 \\
\hline $\mathrm{SBP}, \mathrm{mmHg}$ & $147 \pm 8$ & $148 \pm 7$ & 0.653 \\
\hline $\mathrm{DBP}, \mathrm{mmHg}$ & $80 \pm 9$ & $75 \pm 12$ & 0.077 \\
\hline Heart rate, $/ \mathrm{min}$ & $73 \pm 11$ & $74 \pm 11$ & 0.773 \\
\hline $\mathrm{PP}, \mathrm{mmHg}$ & $67 \pm 12$ & $72 \pm 12$ & 0.094 \\
\hline Hematocrit, \% & $42.7 \pm 5.1$ & $41.0 \pm 3.3$ & 0.163 \\
\hline Sodium, mEq/L & $141 \pm 2$ & $141 \pm 2$ & 0.931 \\
\hline Potassium, $\mathrm{mEq} / \mathrm{L}$ & $4.0 \pm 0.4$ & $4.2 \pm 0.3$ & 0.065 \\
\hline Chloride, $\mathrm{mEq} / \mathrm{L}$ & $104 \pm 2$ & $103 \pm 2$ & 0.433 \\
\hline Uric acid, mg/dL & $5.3 \pm 1.2$ & $4.9 \pm 1.2$ & 0.243 \\
\hline Creatinine, $\mathrm{mg} / \mathrm{dL}$ & $0.7 \pm 0.2$ & $0.7 \pm 0.2$ & 0.447 \\
\hline $\mathrm{HbA1c}, \%$ & $5.4 \pm 0.8$ & $5.6 \pm 1.0$ & 0.339 \\
\hline MDA-LDL, U/L & $108 \pm 34$ & $109 \pm 37$ & 0.890 \\
\hline hs-CRP, $\mu \mathrm{g} / \mathrm{dL}$ & $1,629 \pm 2,679$ & $943 \pm 907$ & 0.213 \\
\hline
\end{tabular}

groups. The PP also significantly decreased after randomization in both groups; however, the changes were not significantly different between the two groups. The expression of the oxidative stress marker, MDA-LDL, and the levels of hsCRP were not significantly different at six months compared with those observed at baseline (Table 2).

In order to exclude the effects of differences in the rate of calcium antagonist use between the two groups, we compared the PP and MDA-LDL values only in the patients who received calcium antagonists. Consequently, the mean PP in the diuretic group was $65 \pm 14 \mathrm{mmHg}$ at baseline and $58 \pm 14 \mathrm{mmHg}$ at six months $(\mathrm{p}=0.161$, vs. $0 \mathrm{~m})$, while that in the non-diuretic group was $71 \pm 12 \mathrm{mmHg}$ at baseline and $60 \pm 11 \mathrm{mmHg}$ at six months $(\mathrm{p}=0.002$, vs. $0 \mathrm{~m} ; \mathrm{p}=0.268$, diuretic vs. non-diuretic group in terms of the changes in the values from 0 to $6 \mathrm{~m}$ ). In contrast, the MDA-LDL values at six months remained unchanged compared with those observed at baseline in both groups: the MDA-LDL values in the diuretic group and the non-diuretic group were 103 \pm $35 \mathrm{U} / \mathrm{L}$ and $111 \pm 38 \mathrm{U} / \mathrm{L}$ at baseline, respectively, and $118 \pm$ $54 \mathrm{U} / \mathrm{L}(\mathrm{p}=0.327$, vs. $0 \mathrm{~m}$ ) and $107 \pm 38 \mathrm{U} / \mathrm{L}$ ( $\mathrm{p}=0.728$, vs. 0 $\mathrm{m} ; \mathrm{p}=0.143$, diuretic vs. non-diuretic group in terms of the changes in the values from 0 to $6 \mathrm{~m}$ ) at six months, respectively.

\section{Discussion}

In the present study, the addition of HCTZ to losartan did not result in any further evident antioxidant effects and the degree of PP reduction obtained with diuretics was not prominent compared with that achieved with non-diuretic antihypertensive drugs.

Pivotal clinical trials of antihypertensive treatment have shown that thiazide diuretics induce significant reductions in the risk of cardiovascular events $(1,2)$. Previous studies have also suggested that thiazide diuretics have antioxidant effects, which may contribute to the beneficial effects of these drugs noted in patients with cardiovascular disease $(11,12)$. Although we hypothesized that HCTZ has more potent antioxidant effects than other non-diuretic anti- 
Table 2. Changes in Blood Pressure, Heart Rate and Serum Parameters after Treatment with Diuretic or Non-diuretic Antihypertensive Drugs

\begin{tabular}{|c|c|c|c|c|c|c|c|}
\hline & \multicolumn{3}{|c|}{ Diuretic group } & \multicolumn{3}{|c|}{ Non-diuretic group } & \multirow[b]{2}{*}{$\mathrm{p}^{\dagger}$} \\
\hline & $0 \mathrm{~m}$ & $6 \mathrm{~m}$ & $\mathrm{p}^{*}$ & $0 \mathrm{~m}$ & $6 \mathrm{~m}$ & $\mathrm{p}^{*}$ & \\
\hline $\mathrm{SBP}, \mathrm{mmHg}$ & $147 \pm 8$ & $133 \pm 12$ & $<0.001$ & $148 \pm 7$ & $134 \pm 10$ & $<0.001$ & 0.911 \\
\hline DBP, $\mathrm{mmHg}$ & $80 \pm 9$ & $75 \pm 10$ & 0.015 & $75 \pm 11$ & $73 \pm 8$ & 0.157 & 0.265 \\
\hline Heart rate, /min & $73 \pm 11$ & $72 \pm 10$ & 0.791 & $74 \pm 11$ & $74 \pm 10$ & 0.886 & 0.886 \\
\hline $\mathrm{PP}, \mathrm{mmHg}$ & $67 \pm 12$ & $58 \pm 12$ & $<0.001$ & $72 \pm 12$ & $61 \pm 12$ & $<0.001$ & 0.452 \\
\hline Hematocrit, \% & $42.7 \pm 5.1$ & $42.1 \pm 5.4$ & 0.330 & $41.0 \pm 3.3$ & $40.9 \pm 3.3$ & 0.843 & 0.583 \\
\hline Sodium, $\mathrm{mEq} / \mathrm{L}$ & $141 \pm 2$ & $140 \pm 4$ & 0.563 & $141 \pm 2$ & $140 \pm 2$ & 0.217 & 0.985 \\
\hline Potassium, $\mathrm{mEq} / \mathrm{L}$ & $4.0 \pm 0.4$ & $4.0 \pm 0.3$ & 0.594 & $4.2 \pm 0.3$ & $4.1 \pm 0.3$ & 0.181 & 0.416 \\
\hline Chloride, $\mathrm{mEq} / \mathrm{L}$ & $104 \pm 2$ & $102 \pm 4$ & 0.042 & $103 \pm 2$ & $104 \pm 2$ & 0.656 & 0.056 \\
\hline Uric acid, mg/dL & $5.3 \pm 1.2$ & $6.0 \pm 1.0$ & $<0.001$ & $4.9 \pm 1.2$ & $4.8 \pm 1.3$ & 0.503 & $<0.001$ \\
\hline Creatinine, $\mathrm{mg} / \mathrm{dL}$ & $0.7 \pm 0.2$ & $0.8 \pm 0.2$ & 0.211 & $0.7 \pm 0.2$ & $0.7 \pm 0.2$ & 0.505 & 0.590 \\
\hline HbAlc, $\%$ & $5.4 \pm 0.8$ & $5.6 \pm 0.7$ & 0.008 & $5.6 \pm 1.0$ & $5.7 \pm 0.9$ & 0.205 & 0.421 \\
\hline MDA-LDL, U/L & $108 \pm 34$ & $117 \pm 54$ & 0.259 & $109 \pm 37$ & $105 \pm 39$ & 0.596 & 0.237 \\
\hline Log hs-CRP, $\mu \mathrm{g} / \mathrm{dL}$ & $2.87 \pm 0.51$ & $2.95 \pm 0.49$ & 0.272 & $2.81 \pm 0.38$ & $2.87 \pm 0.42$ & 0.475 & 0.852 \\
\hline
\end{tabular}

hypertensive drugs, the superiority of HCTZ in terms of its antioxidant effects was not evident in this study. One possible reason for this finding is that the antioxidant effects of different thiazide diuretics might not be the same. For example, chlorthalidone and indapamide have been shown to have more potent antioxidant effects than other thiazides $(11,12)$. Another report showed that HCTZ does not significantly decrease the erythrocyte MDA concentrations in hypertensive patients (13). Another reason may be that antihypertensive drugs, such as ARBs, calcium antagonists, $\beta$-blockers and $\alpha$-blockers, themselves have an antioxidant effect (14-17) that possibly obscures the antioxidant effects of thiazide diuretics. Since the rate of calcium antagonist use differed between the two groups in the current study, we additionally performed a sensitivity analysis of the patients who received calcium antagonists. However, the results remained unchanged, indicating that the different rate of calcium antagonist use did not affect the outcomes. Based on these findings, the antioxidant effects of HCTZ may be minor.

The PP is a strong independent predictor of cardiovascular risk in hypertensive patients. A previous report demonstrated that HCTZ reduces the PP values more significantly than captopril, atenolol, clonidine, diltiazem or prazosin after one year of treatment (6). Considering these data, HCTZ is expected to reduce the PP more potently than non-diuretic antihypertensive drugs. In the present study, both the HCTZ and non-diuretic groups showed a decrease in PP after treatment; however, no significant differences were observed between the two groups. The mechanism of PP reduction is thought to involve the normalization of arterial stiffness and wall thickness (18). A longer observation period may be needed to detect morphological changes in the vessel wall with subsequent reductions in $\mathrm{PP}$, as previous studies have mostly observed such effects after one year $(5,6)$.

Previous reports have also shown that combination treatment with losartan/HCTZ safely and steadily reduces the BP (19-21) and improves the quality of life in patients with uncontrolled hypertension (21). Furthermore, in patients with symptomatic internal carotid artery or middle cerebral artery steno-occlusive disease, this drug regimen also safely lowers the BP without worsening the cerebral hemodynamics (22). In the current study, no adverse events were observed over six months of treatment in the losartan/HCTZ group. A longer follow-up period may be needed in order to assess the safety of losartan/HCTZ in chronic stroke patients.

The present study is associated with some limitations. First, the sample size was small. Patient recruitment was likely stopped before the number of enrolled patients reached a sufficient sample size. Although it was difficult to estimate the mean and standard deviation of the PP and MDL-LDL before the start of the study, the required sample size is expected to be at least twice the size of that used in the present study in order to detect differences in PP and MDA-LDL with $80 \%$ statistical power and a level of significance of $5 \%$. Therefore, the possibility of a type I or II error cannot be excluded. Furthermore, other confounding factors that may alter the degree of oxidative stress, such as smoking and vitamin supplementation, were not evaluated, and we measured only the level of MDL-LDL as an oxidative stress marker. Although the MDL-LDL level is a commonly used and reliable marker, there are many useful oxidative stress markers. The use of measurements of other oxidative stress markers, such as 8-iso-prostaglandin F2 $\alpha$ (8iso-PGF2 $\alpha$ ), 8-hydroxy-2'-deoxyguanosine (8-OHdG) and thiobarbituric acid reactive substances (TBARS), and adjustment for possible confounding factors related to oxidative stress would have strengthened our conclusions. Since the present study provided preliminary results based on data for a limited number of patients, further studies with larger sample sizes are thus needed to clarify the additional effects of losartan/HCTZ beyond the blood pressure-lowering actions of these drugs. 


\section{Author's disclosure of potential Conflicts of Interest (COI).} Takanari Kitazono: Honoraria, Eizai, MSD, Daiichi-Sankyo, Takeda Pharmaceutical, Mitsubishi Tanabe Pharma, Nippon Boeringer Ingelhaim, Novartis Pharma, Bayer Yakuhin and Pfizer Japan; Research funding: Mitsubishi Tanabe Pharma, Takeda Pharmaceutical, Eizai, MSD, Daiichi-Sankyo, Pfizer Japan, Nippon Boeringer Ingelhaim, Novartis Pharma, Chugai Pharmaceutical, Astellas Pharma, Otsuka Pharmaceutical, Kyowa Hakko Kirin and Sanofi.

\section{Acknowledgement}

We thank all patients who participated in this study and all collaborators and clinical research coordinators for their help in obtaining informed consent and collecting the clinical data.

This work was supported by MSD KK. MSD supported meetings with the steering committee. The steering committee included H. Nakane, M. Kamouchi, S. Ibayahsi, K. Kusuda, T. Omae, T. Nagao and T. Kitazono.

\section{References}

1. ALLHAT Officers and Coordinators for the ALLHAT Collaborative Research Group. The ANtihypertensive and Lipid-Lowering Treatment to Prevent Heart Attack Trial. Major outcomes in highrisk hypertensive patients randomized to angiotensin-converting enzyme inhibitor or calcium channel blocker vs diuretic: The Antihypertensive and Lipid-Lowering Treatment to Prevent Heart Attack Trial (ALLHAT). JAMA 288: 2981-2997, 2002.

2. Prevention of stroke by antihypertensive drug treatment in older persons with isolated systolic hypertension. Final results of the Systolic Hypertension in the Elderly Program (SHEP). SHEP Cooperative Research Group. JAMA 265: 3255-3264, 1991.

3. de Champlain J, Wu R, Girouard H, et al. Oxidative stress in hypertension. Clin Exp Hypertens 26: 593-601, 2004.

4. Miyagawa $\mathrm{K}$, Ohashi $\mathrm{M}$, Yamashita $\mathrm{S}$, et al. Increased oxidative stress impairs endothelial modulation of contractions in arteries from spontaneously hypertensive rats. J Hypertens 25: 415-421, 2007.

5. Asmar RG, London GM, O'Rourke ME, Safar ME, Coordinators $\mathrm{RP}$, Investigators. Improvement in blood pressure, arterial stiffness and wave reflections with a very-low-dose perindopril/indapamide combination in hypertensive patient: a comparison with atenolol. Hypertension 38: 922-926, 2001.

6. Cushman WC, Materson BJ, Williams DW, Reda DJ. Pulse pressure changes with six classes of antihypertensive agents in a randomized, controlled trial. Hypertension 38: 953-957, 2001.

7. Julius S, Kjeldsen SE, Weber M, et al; Value trial group. Outcomes in hypertensive patients at high cardiovascular risk treated with regimens based on valsartan or amlodipine: the VALUE randomised trial. Lancet 363: 2022-2031, 2004.

8. Ogihara T, Nakao K, Fukui T, et al; Candesartan Antihypertensive Survival Evaluation in Japan Trial, Group. Effects of candesartan compared with amlodipine in hypertensive patients with high cardiovascular risks: candesartan antihypertensive survival evaluation in Japan trial. Hypertension 51: 393-398, 2008.
9. Ogihara T, Kikuchi K, Matsuoka H, et al; Japanese Society of Hypertension Comittee. The Japanese Society of Hypertension Guidelines for the Management of Hypertension (JSH 2009). Hypertens Res 32: 3-107, 2009.

10. Mancia G, De Backer G, Dominiczak A, et al; ESH-ESC Task Force on the Management of Arterial Hypertension. 2007 ESHESC Practice Guidelines for the Management of Arterial Hypertension: ESH-ESC Task Force on the Management of Arterial Hypertension. J Hypertens 25: 1751-1762, 2007.

11. Uehara Y, Kawabata Y, Shirahase H, et al. Oxygen radical scavengers and renal protection by indapamide diuretic in salt-induced hypertension of dahl strain rats. J Cardiovasc Pharmacol 22 Suppl 6: S42-S46, 1993.

12. Sato K, Dohi Y, Kojima M, Takase H, Suzuki S, Ito S. Antioxidative effects of thiazide diuretics in refractory hypertensive patients. Arzneimittelforschung 60: 612-616, 2010.

13. Kedziora-Kornatowska K, Czuczejko J, Szewczyk-Golec K, et al. Effects of perindopril and hydrochlorothiazide on selected indices of oxidative stress in the blood of elderly patients with essential hypertension. Clin Exp Pharmacol Physiol 33: 751-756, 2006.

14. Pialoux V, Foster GE, Ahmed SB, Beaudin AE, Hanly PJ, Poulin MJ. Losartan abolishes oxidative stress induced by intermittent hypoxia in humans. J Physiol 589: 5529-5537, 2011.

15. Lupo E, Locher R, Weisser B, Vetter W. In vitro antioxidant activity of calcium antagonists against LDL oxidation compared with alpha-tocopherol. Biochem Biophys Res Commun 203: 18031808, 1994.

16. Maggi E, Marchesi E, Covini D, Negro C, Perani G, Bellomo G. Protective effects of carvedilol, a vasodilating beta-adrenoceptor blocer, against in vivo low density lipoprotein oxidation in essential hypertension. J Cardiovasc Pharmacol 27: 532-538, 1996.

17. Jansen H, Lammers R, Baggen MG, Birkenhäger JC. Effects of doxazosin on lipids, lipoprotein lipases, and cholesterol synthesis in the golden hamster. J Cardiovasc Pharmacol 13 Suppl 2: S5-S9, 1989.

18. Safar ME, van Bortel LM, Struijker-Boudier HA. Resistance and conduit arteries following converting enzyme inhibition in hypertension. J Vasc Res 34: 67-81, 1997.

19. Kita T, Yokota N, Ichiki Y, et al. Three-year safety and effectiveness of fixed-dose losartan/hydrochlorothiazide combination therapy in Japanese patients with hypertension under clinical setting (PALM-1 Extension Study). Clin Exp Hypertens 34: 498-503, 2012.

20. Hosoya T, Kuriyama S, Ohno I, et al. Antihypertensive effect of a fixed-dose combination of losartan/hydrochlorothiazide in patients with uncontrolled hypertension: a multicenter study. Clin Exp Nephrol 16: 269-278, 2012.

21. Kamura A, Inoue $T$, Kuroki $S$, et al. Antihypertensive treatment using an angiotensin receptor blocker and a thiazide diuretic improves patients' quality of life: The Saga Challenge Antihypertensive Study (S-CATS). Hypertens Res 34: 1288-1294, 2011.

22. Saura H, Ogasawara K, Suzuki T, et al. Effect of combination therapy with the angiotensin receptor blocker losartan plus hydrochlorothiazide on brain perfusion in patients with both hypertension and cerebral hemodynamic impairment due to symptomatic chronic major cerebral artery steno-occlusive disease: a SPECT study. Cerebrovasc Dis 33: 354-361, 2012.

(C) 2015 The Japanese Society of Internal Medicine http://www.naika.or.jp/imonline/index.html 